\title{
ON THE CONVERGENCE OF MULTIPLICATIVELY ORTHOGONAL SERIES
}

\author{
C. J. PRESTON
}

Abstract. G. Alexits and A. Sharma have recently shown that if $\left\{\varphi_{n}\right\}_{n-1}^{\infty}$ is a uniformly bounded multiplicatively orthogonal system on a finite measure space and if $\left\{c_{n}\right\}_{n-1}^{\infty}$ is a sequence of real numbers with $\sum_{n=1}^{\infty} c_{n}^{2}<\infty$, then the partial sums $\sum_{k=1}^{n} c_{k} \varphi_{k}$ converge almost everywhere. We give here a simple proof of this result.

Let $(X, \beta, \mu)$ be a measure space, with $\mu$ a finite nonnegative measure, and let $f_{n}: X \rightarrow R, n=1,2, \cdots$, be an orthonormal system on $(X, B, \mu)$, (i.e. $f_{n} \in L^{2}(X, @, \mu)$ with $\left.\int_{X} f_{n} f_{m} d \mu=\delta_{m, n}\right)$. Let $c_{n}$ $\in R, n=1,2, \cdots$, and define $s_{n}$ by $s_{n}(x)=\sum_{\nu=1}^{n} c_{\nu} f_{\nu}(x)$. Then the classical result of Menchoff states that $s_{n}$ converges a.e. as $n \rightarrow \infty$, provided $\sum_{n=1}^{\infty} c_{n}^{2}(\log n)^{2}<\infty$. Menchoff also showed that for a general orthonormal system this is the best result possible. For particular orthonormal systems we can get better results; for example, if $X=T$, and $\mu=$ Lebesgue measure on $T$, and $f_{n}(x)=\cos n x$, or $f_{n}(x)=\sin n x$, then it follows from the famous result of Carleson that $s_{n}$ converges a.e. as $n \rightarrow \infty$ provided $\sum_{n=1}^{\infty} c_{n}^{2}<\infty$. In a preprint of a paper to appear in Acta. Math. Acad. Sci. Hungar., G. Alexits and A. Sharma prove a similar result for uniformly bounded multiplicatively orthogonal systems. (We say $\left\{\varphi_{n}\right\}_{n=1}^{\infty}$ is a uniformly bounded multiplicatively orthogonal system on $(X, ß, \mu)$ if $\varphi_{n} \in L^{\infty}(X, ß, \mu)$ with $\left\|\varphi_{n}\right\|_{\infty} \leqq M$ for some $M$ and all $n$, and if given any $m=1,2, \cdots$, and $1 \leqq \nu_{1}<\nu_{2}<\cdots<\nu_{m}$, then $\int_{X} \varphi_{\nu_{1}} \cdots \varphi_{\nu_{m}} d \mu=0$.)

Alexits and Sharma prove the following:

ThEOREM. Let $\left\{\varphi_{n}\right\}_{n=1}^{\infty}$ be a uniformly bounded multiplicatively orthogonal system on $(X, \Theta, \mu)$. Let $c_{n} \in R, n=1,2, \cdots$, and let $s_{n}(x)$ $=\sum_{\nu=1}^{n} c_{\nu} \varphi_{\nu}(x)$. Then $s_{n}$ converges a.e. as $n \rightarrow \infty$ provided $\sum_{n=1}^{\infty} c_{n}^{2}<\infty$.

The proof of this theorem by Alexits and Sharma involves some difficult constructions; we give here a short and simple proof.

We may suppose without loss of generality that $\left|\varphi_{n}(x)\right| \leqq 1$ for all $x \in X$ and for all $n$. Let $\left\{\psi_{n}\right\}_{n=0}^{\infty}$ be the product system associated with $\left\{\varphi_{n}\right\}_{n=1}^{\infty}$; i.e.

Received by the editors July $27,1970$.

AMS 1970 subject classifications. Primary 40A05, 42A60, 42A20.

Key words and phrases. Uniformly bounded multiplicatively orthogonal systems, almost everywhere convergence. 


$$
\begin{aligned}
& \psi_{n}=\varphi_{v_{1}+1} \cdots \varphi_{v_{m}+1} \text { for } n=2^{v_{1}}+\cdots+2^{v_{m}}, \\
& \psi_{0} \equiv 1 .
\end{aligned}
$$

Note the following two facts:

$$
\int_{X} \psi_{n} d \mu=0, \quad n=1,2, \cdots ;
$$

(2) $\quad \sum_{k=0}^{2^{m}-1} \psi_{k}(x) \psi_{k}(y)=\prod_{k=1}^{m}\left(1+\varphi_{k}(x) \varphi_{k}(y)\right) \geqq 0 \quad$ for all $x, y \in X$.

Define $n(x)$ to be the least index such that $s_{n(x)}(x)=\max _{1 \leq \nu \leq n} s_{\nu}(x)$. We have

$$
s_{n}(x)=\sum_{k=0}^{2^{n}-1} a_{k} \psi_{k}(x)
$$

where

$$
\begin{aligned}
a_{k} & =c_{\nu+1} & & \text { if } k=2^{\nu}, \\
& =0 & & \text { otherwise, }
\end{aligned}
$$

and so $s_{n(x)}(x)=\sum_{k=0}^{2^{n(x)}-1} a_{k} \psi_{k}(x)$.

Let $(Y, a, \omega)$ be any finite measure space, and let $\left\{g_{n}\right\}_{n=0}^{\infty}$ be any orthonormal system on $(Y, a, \omega)$. Then

$$
S_{n(x)}(x)=\int \sum_{Y=0}^{2^{n}-1} a_{k} g_{k}(t) \sum_{j=0}^{2^{n}(x)} \psi_{j}(x) g_{j}(t) d \omega(t) .
$$

Therefore

$$
\begin{aligned}
&\left|\int_{X} s_{n(x)}(x) d \mu(x)\right|=\left|\int_{Y} \sum_{k=0}^{2^{n}-1} a_{k} g_{k}(t) \int_{X} \sum_{j=0}^{2^{n}(x)} \psi_{j}(x) g_{j}(t) d \mu(x) d \omega(t)\right| \\
& \leqq\left\{\int_{Y}\left[\sum_{k=0}^{2^{n}-1} a_{k} g_{k}(t)\right]^{2} d \omega(t)\right. \\
&\left.\cdot \int_{Y}\left[\int_{X} \sum_{k=0}^{2^{n(x)}-1} \psi_{k}(x) g_{k}(t) d \mu(x)\right]^{2} d \omega(t)\right\}^{1 / 2} \\
&=\left\{\left(\sum_{k=0}^{2^{n}-1} a_{k}^{2}\right) \int_{Y} \int_{X} \int_{X}^{2^{n}(x)-1} \sum_{k=0}^{\psi_{k}} \psi_{k}(x) g_{k}(t)\right. \\
&\left.\cdot \sum_{j=0}^{2^{n}(y)} \psi_{j}(y) g_{j}(t) d \mu(x) d \mu(y) d \omega(t)\right\}^{1 / 2}
\end{aligned}
$$


Thus

$$
\begin{aligned}
& \left|\int_{X} s_{n(x)}(x) d \mu(x)\right|^{2} \\
& \leqq\left(\sum_{k=1}^{n} c_{k}^{2}\right) \int_{X} \int_{X} \int_{Y} \sum_{k=0}^{2^{n(x)}-1} \psi_{k}(x) g_{k}(t) \sum_{j=0}^{2^{n(y)}-1} \psi_{j}(y) g_{j}(t) d \omega(t) d \mu(x) d \mu(y) \\
& =\left(\sum_{k=1}^{n} c_{k}^{2}\right) \int_{X} \int_{X} \sum_{k=0}^{2^{n}(x, y)-1} \psi_{k}(x) \psi_{k}(y) d \mu(x) d \mu(y), \\
& \leqq\left. 2\left(\sum_{k=1}^{n} c_{k}^{2}\right) \int_{X} \int_{X}\right|^{2^{n(y)}-1} \sum_{k=0} \psi_{k}(x) \psi_{k}(y) \mid d \mu(x) d \mu(y) \\
& =2\left(\sum_{k=1}^{n} c_{k}^{2}\right) \int_{X} \int_{X}^{2^{n}(y)-1} \sum_{k=0}^{n} \psi_{k}(x) \psi_{k}(y) d \mu(x) d \mu(y) \quad \text { where } n(x, y)=\min \{n(x), n(y)\} \\
& \left.=2\left(\sum_{k=1}^{n} c_{k}^{2}\right) \int_{X} \int_{X} \psi_{0}(x) \psi_{0}(y) d \mu(x) d \mu(y) \quad \text { (using (1)) }\right) \\
& =2\left(\sum_{k=1}^{n} c_{k}^{2}\right)[\mu(X)]^{2} .
\end{aligned}
$$

Hence we have

$$
\left|\int_{X} s_{n(x)}(x) d \mu(x)\right|^{2} \leqq 2\left(\sum_{k=1}^{\infty} c_{k}^{2}\right)[\mu(X)]^{2} .
$$

It is well known that such an estimate is sufficient in order to prove the theorem.

Corneli. University, Ithaca, New York 14850 